\title{
Evaluation of favipiravir treatment before intensive care in COVID-19 patients
}

\section{Osman Ozudogru $\square \mathbb{O}^{1}$, Emrah Yerlikaya $\square \mathbb{C}^{2}$, Naci Omer Alayunt
$\mathbb{1}^{3}$ Zafer Cambay $\square{ }^{4^{*}}$}

Department of Internal Medicine, Siirt Training and Research Hospital, Siirt, Turkey

2 Department of Nutrition and Dietetics, Faculty of Health Sciences, Siirt University, Siirt, Turkey

3 Department of Medical Biochemistry, Faculty of Medicine, Siirt University, Siirt, Turkey

4 Firat University Health Services Vocational School, Elazig, Turkey

\begin{abstract}
The purpose of this study is to investigate the healing effect of favipiravir used in pre-intensive care treatment of patients diagnosed with COVID-19 in order to elucidate the pathogenesis and complications of coronavirus. The data regarding the clinical findings of the patients in the hospital information system and the biochemical parameters made standard in the treatment or follow-up of COVID 19 were taken from the system and evaluated retrospectively. In addition, it was examined as a whole with mild, moderate, and severe pulmonary involvement compared to CT findings. Hemogram, coagulation, and biochemistry parameters used in the diagnosis and follow-up of COVID19 were evaluated. SPSS 22.0 statistics program for Windows was used in statistical analysis to evaluate the data obtained from patient files and hospital information systems. There is no standard treatment protocol within the scope of treatment. Drug studies are currently ongoing. In this study, the first clinical findings, treatment types, and recovery times of patients diagnosed with COVID-19, the healing effect of favipiravir used before intensive care were determined. Between-group 1 (those who started treatment within 0-5 days) and group 2 (those who started treatment within 6-10 days), after five days of favipiravir treatment, when serum parameters were compared, favipiravir treatment was statistically significantly lower in the first group that was started early, WBC, Neutrophil, Creatine, CK, CRP, D-Dimer, PCT, LDH. By collecting the data obtained as a result of the research, early deaths can be prevented worldwide. Our study recommending alternative treatment approaches is vital for the protection of patients' quality of life. In this study, when all biochemical markers were evaluated together, it was evaluated that starting Favipiravir treatment early was beneficial in treating COVID-19 disease.
\end{abstract}

Keywords: Coronavirus; Favipiravir; Covid-19; Intensive Care; Pandemic.

\section{INTRODUCTION}

Antiviral drug development is determined by the viral life cycle through viral replication stages and cellular processes that support viral replication. The effect of antivirals targeting a viral replication step can be enhanced by causing synergies with secondary effects through an antiviral or drug metabolism that hits a different viral target or a cell process. The stages of antiviral drug development begin in silico design. They are tested in single-cell types (organotypic cell lines or primary cells) and animal models, clinical trials, and ultimately regulatory approval/market complex models to determine $I_{50} / C_{50}=S I$. A significant obstacle in developing antivirals is that many compounds that exhibit in vitro 
activity have little effect in animal models. Human organoids/complex in vitro infection models (e.g., Barrier models) can provide a bridge to predict activity in clinical trials. It is well known that even the threat of biological attack will cause mass hysteria accompanying economic cuts. Only visible preparation with visible infrastructure, drug and vaccine stocks, and well-thought-out emergency plans will allow governments to provide the necessary assurances when necessary to prevent negative consequences. ${ }^{-}$While many virus infections are asymptomatic, new or improved antiviral drugs are needed to prevent and/or treat several important conditions caused by viruses that cannot be controlled by alternative measures, including vector control, immunization, and current antiviral therapy. Antiviral compounds that are effective in infections caused by tropical and vector viruses have been a neglected subject of international antiviral research until recently.

Some compounds are currently in clinical trials, and few have regulatory approval or are available on the market. FDA approval is pending for the use of favipiravir to treat filovirus infections. Several animal pilot studies in non-human primates (NHP) have recently demonstrated the efficacy of favipiravir. $\stackrel{\text { Toyama }}{\text { Th }}$ Chemical Co., Ltd. Favipiravir (T-705), discovered and synthesized, shows a wide range of activities against influenza virus, arenaviruses, bunyaviruses, West Nile virus, foot and mouth disease virus, yellow fever virus, and various RNA viruses. ${ }^{3}$ Favipiravir is converted to its active form, ribofuranosyl-5-triphosphate, by host enzymes and inhibits viral RNA polymerase in host cells. Only a few reports have resisted favipiravir in vitro., 4 In research conducted by Janowski $A$. et al. on the treatment of favipiravir in 2020, Astrovirus VA1 (VA1) replication had a 10 -fold reduction.

However, only a $44 \%$ reduction in human astrovirus 4 (HAstV4) replication was observed at $1000 \mu \mathrm{M} .^{6}$ No significant decrease in cell viability was observed in any favipiravir concentration. The results show that favipiravir inhibits VA1 replication. The in vitro $\mathrm{EC}_{50}$ values defined for the drug have similar activities for other enteric viruses such as enteroviruses and noroviruses. Favipiravir is also used in the treatment of Thrombocytopenia Syndrome with Severe Fever (SFTS). Studies of potential antiviral drugs for SFTS-specific therapy have been performed on existing or newly discovered agents in vitro and in vivo. Ribavirin and favipiravir are the most promising candidates. Although animal experiments and retrospective studies have proven the limited efficacy of ribavirin, ribavirin is thought to be effective in patients with a viral load of $<1 \times 10^{6} \mathrm{copies} / \mathrm{mL}$.

Favipiravir showed higher efficacy than ribavirin against SFTS in vitro experiments. Higher efficacy was observed in animal models administered even three days after virus inoculation. ${ }^{?}$ In vivo efficacy of favipiravir has been studied using animal models. Intraperitoneal (i.p.) administration of favipiravir at a dose of 60 or $300 \mathrm{mg} / \mathrm{kg} /$ day for five days ensured that mice were fully protected from death upon SFTS infection. On the other hand, $40 \%$ of mice treated with ribavirin (i.p.) at a dose of 25 or $100 \mathrm{mg} / \mathrm{kg} / \mathrm{day}$ lost body weight and died from SFTS infection, reducing the rate of case death. All mice treated with favipiravir survived when treatment was initiated three days after infection or earlier, whereas mice treated 4 and 5 days after infection exhibited $83 \%$ and $50 \%$ survival, respectively. Generally, favipiravir is administered orally to humans as in the mouse model. $\underline{-}$ In addition, it may be desirable to use intravenous administration as SFTS patients with severe symptoms may have difficulty taking oral medications. ${ }^{7}$ Favipiravir also has a protective effect against Nipah virus infections in the hamster model ${ }^{9}$ and inhibits some paramyxoviruses in vitro. ${ }^{10}$ Favipiravir resistance mechanism has been described in the influenza virus. ${ }^{5}$ 


\section{MATERIAL AND METHOD}

Preliminary permits for the feasibility of the study were obtained from the Public Health Directorate before the start of the study. This study was approved ethically by the decision of Siirt University Non-Interventional Clinical Research Ethics Committee dated 02/06/2020 and number 08.02. In this study, patients who applied to Siirt State Hospital who complained of high fever, difficulty breathing, and weakness were diagnosed with COVID-19 and were administered Favirpiravir before intensive care were examined. The patient groups consisted of those who started treatment up to the first five days after hospitalization and started treatment after five days in the second group. That is, the first group was determined as those who started treatment within the first five days after hospitalization (together with favipiravir hydroxychloroquine + azithromycin + oseltamivir), and the second group (hydroxychloroquine + azithromycin + oseltamivir before the favipiravir) (Table 4 and $\underline{5}$ ). According to the science committee's guidelines, the Ministry of Health of the Republic of Turkey has been moving. The groups were compared among themselves. Hemogram, coagulation, and biochemistry parameters (Ferritin, Creatine, CK, CRP, D-Dimer, PCT, LDH) were used to diagnose, and follow-up of COVID-19 was evaluated. SPSS 22.0 statistics program for Windows was used in statistical analysis to evaluate the data obtained from patient files and hospital information systems. The biochemical parameters of patients were also evaluated retrospectively. In the biochemistry laboratory, biochemical parameters were studied using Siemens Dimension RxL Max Integrated Chemistry System.

Statistical analyzes using chi-square to show the differences between the time of starting the drug and demographic data. In cases where the expected value was below 5, chi-square Fisher's Exact Test was used. Spearman correlation test was used to determine the relationship between the data. Independent t-Test was used to determine the significance of differences between the time to start medication and biochemical parameters.

\section{RESULTS AND DISCUSSION}

When comparing demographic data, chronic diseases, smoking, tomography involvement, and initial symptoms between group 1 (those who started treatment in 0-5 days) and group 2 (those who started treatment in 6-10 days), no statistically significant difference was found. (Table 1). Serum parameters were evaluated between group 1 (those who started treatment within 0-5 days) and group 2 (those who started treatment within 6-10 days) at the time of treatment, CRP and D-Dimer values were in group 1; Although CK, LDH and Ferritin values were higher in the 2nd group, no statistically significant difference was found between the two groups (Table 2). Serum WBC, neutrophils, creatine, CK, CRP, D-Dimer, PCT, and LDH levels of patients whose drug start time is 0-5 days were statistically significantly different (Table 3 ) compared to the patients whose drug start time is $6-10$ days serum levels $(p<0.05)$. Between-group 1 (those who started treatment within 0-5 days) and group 2 (those who started treatment within 6-10 days), after five days of favipiravir treatment (Table 4), when serum parameters were compared, favipiravir treatment was statistically significantly lower in the first group that was started early, WBC, Neutrophil, Creatine, CK, CRP, D-Dimer, PCT, LDH. It is crucial that CRP, D-Dimer markers, which are stated as harmful prognostic factors, especially in covid-19 disease, are lower than the second group and may indicate a better clinical response if treatment is started early. Ferritin value, a prognostic marker, was found lower in the first group but was not statistically significant. When the lymphocyte values, one of the diagnostic criteria and prognostic markers, were examined, it was higher in the first group but not statistically significant ( $\underline{\text { Table 3 }}$ ). 
In order to elucidate the pathogenesis and complications of coronavirus, the healing effect of favipiravir used in pre-intensive care treatment of patients diagnosed with COVID-19 was investigated. It has also been compared with its applications during intensive care in the world. Republic of Turkey Ministry of Health Sciences Board has published numerous guidelines during the pandemic. As can be seen in the guides, While favipiravir treatment was recommended to intubated (critical) patients in intensive care at the beginning of the pandemic, it was recommended to start before intensive care in patients with moderatesevere pneumonia / non-responding to the first-line treatment (hydroxychloroquine-azithromycin-oseltamivir) in the following guides. Favipiravir was approved for the new flu treatment on February 15, 2020, in China, but clinical trials are ongoing to treat COVID-19. Favipiravir is a new type of RNAdependent RNA polymerase (RdRp) inhibitor. In addition to its anti-influenza virus activity, favipiravir can block the replication of flavi-, alpha-, filo-, bunya-, arena-, noro- and other RNA viruses. ${ }^{11}$ Favipiravir is converted to an active phosphorylated form in cells and recognized as a substrate by viral RNA polymerase, thereby inhibiting RNA polymerase activity. ${ }^{12}$ Therefore, it was thought that favipiravir might have a potential antiviral effect on SARS-CoV-2, an RNA virus.

On February 14, a clinical trial on favipiravir for the treatment of COVID19, initiated by the National Infectious Diseases Clinical Medical Research Center and Shenzhen Third People's Hospital, had promising results. Initial results of a total of 80 patients (including the experimental group and the control group) showed that favipiravir had a more substantial antiviral effect than lopinavir+ritonavir. ${ }^{12}$ There were no significant adverse reactions in the favipiravir treatment group and significantly fewer adverse events than the lopinavir+ritonavir group..$^{13}$ Favipiravir has complex, nonlinear, time- and dosedependent pharmacokinetics influenced by weight. ${ }^{14,15}$ Because favipiravir is metabolized and inhibited by aldehyde oxidase, initial oral loading is required to obtain adequate blood levels, and the plasma half-life is 4 hours. In persons with hepatic dysfunction, blood concentration should be monitored and the dose adjusted. Favipiravir or its metabolites have been detected in semen and breast milk. Although favipiravir showed no pharmacokinetic interaction with oseltamivir, overexposure to acetaminophen was observed when coadministered with acetaminophen in healthy volunteers..$^{14,15}$ When used together with acetaminophen (paracetamol), the dose of acetaminophen should not exceed $3000 \mathrm{mg} / \mathrm{day}$ (less than in liver failure). When used together with theophylline, the possibility of side effects increases as the blood concentration of the drug increases. Therefore, caution should be exercised when using them together. ${ }^{16,17,18}$ The most common side effects are diarrhea, increase in serum uric acid level, increase in serum transaminase (ALT, AST, ALP) and total bilirubin levels, and decrease in neutrophil levels. Attention should be paid to the use of the drug. $\frac{18}{}$

In a study by Janowski et al., the $\mathrm{EC}_{50}$ values for ribavirin and favipiravir were quantified against two human astrovirus strains, astrovirus VA1 (VA1) and human astrovirus 4 (HAstV4). VA1 replication was inhibited 10-100-fold by both ribavirin $\left(E C_{50}=154 \mu \mathrm{M}\right)$ and favipiravir $\left(E C_{50}=246 \mu \mathrm{M}\right)$. In contrast, ribavirin inhibited HAstV4 replication $\left(E_{50}=268 \mu \mathrm{M}\right)$, but favipiravir only reduced replication by $44 \%$ at the highest dose..$^{6}$ In a study by Tani et al., Favipiravir exhibited higher effectiveness than ribavirin in vitro and in vivo studies. ${ }^{-}$In a study by Yamada et al., Favipiravir (T-705), a broadspectrum antiviral drug against RNA viruses, is effective against Rabies virus in vitro but ineffective in vivo. ${ }^{19}$ These data suggest that favipiravir is a promising drug for the treatment of infections by a specific virus and a wide range of RNA viruses. Favipiravir is efficacious in multiple types of Influenza viruses, regardless of sensitive or 
resistance to existing anti-influenza drugs. A specific note is that favipiravir is active against a wide range of other RNA viruses in vitro and in vivo. In vitro studies indicate no emergence of resistance to favipiravir. ${ }^{20}$ Favipiravir with these unique profiles will be a promising therapeutic agent for unremedied infections by RNA viruses in the near future.

\section{CONCLUSION}

Evidence to date from clinical studies shows that favipiravir is well tolerated in humans. By collecting the data obtained as a result of the research, early deaths can be prevented worldwide. Our study recommending alternative treatment approaches is vital for the protection of patients' quality of life. In this study, when all biochemical markers are evaluated together, it is evaluated that starting Favipiravir treatment early is beneficial in treating COVID-19 disease.

\section{AUTHORS' CONTRIBUTIONS}

All authors contributed equally to this work.

\section{FOUNDING INFORMATION}

This research received no specific grant from any funding agency in public, commercial or not-for-profit sectors.

\section{DATA AVAILABILITY STATEMENT}

The utilized data to contribute to this investigation are available from the corresponding author on reasonable request.

\section{DISCLOSURE STATEMENT}

The views and opinions expressed in this article are those of the authors and do not necessarily reflect the official policy or position of any affiliated agency of the authors.

\section{REFERENCES}

1. Hawley RJ, Eitzen Jr EM. Biological Weapons-a Primer for Microbiologists. Annu Rev Microbiol. 2001;55(1):235-253. doi:10.1146/annurev.micro.55.1.235.

2. Bugert JJ, Hucke F, Zanetta P, Bassetto M, Brancale A. Antivirals in medical biodefense. Virus Genes. 2020;56(2):150-167. doi:10.1007/s11262-020-01737-5.

3. Furuta Y, Takahashi K, Shiraki K, et al. T-705 (favipiravir) and related compounds: Novel broad-spectrum inhibitors of RNA viral infections. Antiviral Res. 2009;82(3):95-102. doi:10.1016/j.antiviral.2009.02.198.

4. Delang L, Segura Guerrero N, Tas A, et al. Mutations in the chikungunya virus non-structural proteins cause resistance to favipiravir (T-705), a broad-spectrum antiviral. J Antimicrob Chemother. 2014;69(10):2770-2784. doi:10.1093/jac/dku209.

5. Goldhill DH, te Velthuis AJW, Fletcher RA, et al. The mechanism of resistance to favipiravir in influenza. Proc Natl Acad Sci. 2018;115(45):11613-11618. doi:10.1073/pnas.1811345115.

6. Janowski $A B$, Dudley $H$, Wang $D$. Antiviral activity of ribavirin and favipiravir against human astroviruses. $J$ Clin Virol. 2020;123:104247. doi:10.1016/j.jcv.2019.104247.

7. Takayama-Ito M, Saijo M. Antiviral Drugs Against Severe Fever With Thrombocytopenia Syndrome Virus Infection. Front Microbiol. 
2020;11. doi:10.3389/fmicb.2020.00150.

8. Tani H, Fukuma A, Fukushi S, et al. Efficacy of T-705 (Favipiravir) in the Treatment of Infections with Lethal Severe Fever with Thrombocytopenia Syndrome Virus. Duprex WP, ed. mSphere. 2016;1(1). doi:10.1128/mSphere.00061-15.

9. Dawes BE, Kalveram B, Ikegami T, et al. Favipiravir (T-705) protects against Nipah virus infection in the hamster model. Sci Rep. 2018;8(1):7604. doi:10.1038/s41598-018-25780-3.

10. Lo MK, Jordan R, Arvey A, et al. GS-5734 and its parent nucleoside analog inhibit Filo-, Pneumo-, and Paramyxoviruses. Sci Rep. 2017;7(1):43395. doi:10.1038/srep43395.

11. Baranovich T, Wong S-S, Armstrong J, et al. T-705 (Favipiravir) Induces Lethal Mutagenesis in Influenza A H1N1 Viruses In Vitro. J Virol. 2013;87(7):3741-3751. doi:10.1128/JVI.02346-12.

12. Kiso M, Takahashi K, Sakai-Tagawa $\mathrm{Y}$, et al. T-705 (favipiravir) activity against lethal H5N1 influenza A viruses. Proc Natl Acad Sci. 2010;107(2):882-887. doi:10.1073/pnas.0909603107.

13. Watanabe T, Kiso M, Fukuyama S, et al. Characterization of H7N9 influenza A viruses isolated from humans. Nature. 2013;501 (7468):551-555. doi:10.1038/nature12392.

14. Madelain V, Nguyen THT, Olivo A, et al. Ebola Virus Infection: Review of the Pharmacokinetic and Pharmacodynamic Properties of Drugs Considered for Testing in Human Efficacy Trials. Clin Pharmacokinet. 2016;55(8):907-923. doi:10.1007/s40262-015-03641 .

15. Nguyen THT, Guedj J, Anglaret $X$, et al. Favipiravir pharmacokinetics in Ebola-Infected patients of the JIKI trial reveals concentrations lower than targeted. Horby PW, ed. PLoS Negl Trop Dis. 2017;11(2):e0005389. doi:10.1371/journal.pntd.0005389.

16. Favipiravir $200 \mathrm{Mg}$ Tablets Prospectus.; 2020.

17. Results D. Avigan Tablet 200 Mg.; 2020.

18. Republic of Turkey Ministry of Health, General Directorate of Public Hospitals, Supply Planning, Stock And Logistics Management Department HPMU. In The Treatment of Covid-19 (SARS-CoV2 Infection) Informatıon on Medications to Be Used.; 2020.

19. Yamada K, Noguchi K, Kimitsuki K, et al. Reevaluation of the efficacy of favipiravir against rabies virus using in vivo imaging analysis. Antiviral Res. 2019;172:104641. doi:10.1016/j.antiviral.2019.104641.

20. Furuta Y, Komeno T, Nakamura T. Favipiravir (T-705), a broad spectrum inhibitor of viral RNA polymerase. Proc Japan Acad Ser B. 2017;93(7):449-463. doi:10.2183/pjab.93.027. 
Table 1: Patients group statistics with demographic data, chronic diseases, smoking, tomography involvement, and initial symptoms.

\begin{tabular}{|c|c|c|c|c|}
\hline & & & art Time & \\
\hline & & $\begin{array}{l}\text { 0-5 days } \\
(\mathrm{n}=28) \\
\%\end{array}$ & $\begin{array}{l}\text { 6-10 days } \\
(\mathrm{n}=8) \\
\%\end{array}$ & $\begin{array}{l}\mathrm{P}^{*} \\
\chi^{2}\end{array}$ \\
\hline & Male & 50 & 75 & $\mathrm{p}=0.257$ \\
\hline Gender & Female & 50 & 25 & $\chi^{2}=1.58$ \\
\hline & + & 21.4 & 37.5 & $p=0.384$ \\
\hline DIVI & - & 78.6 & 62.5 & $\chi^{2}=0.857$ \\
\hline & + & 21.4 & 12.5 & $\mathrm{p}=1.00$ \\
\hline HI & - & 78.6 & 87.5 & $\chi^{2}=0.317$ \\
\hline KBH & - & 100 & 100 & - \\
\hline & + & 17.9 & 0 & $p=0.566$ \\
\hline Caralovascular & - & 82.1 & 100 & $\chi^{2}=1.66$ \\
\hline Pulmonary & + & 14.3 & 12.5 & $\mathrm{p}=1.00$ \\
\hline Disease & - & 85.7 & 87.5 & $\chi^{2}=0.017$ \\
\hline Cigarette & + & 42.9 & 50 & $p=1.00$ \\
\hline & - & 57.1 & 50 & $\chi^{2}=0.129$ \\
\hline Bt & Mild-Middle & 75.7 & 62.5 & $\tilde{p}=0.167$ \\
\hline & Severe & 14.3 & 37.5 & $\chi^{2}=2.141$ \\
\hline Latest Status & Discharged & 96.4 & 87.5 & $p=0.400$ \\
\hline & Ex & 3.6 & 12.5 & $\chi^{2}=0.945$ \\
\hline Clinical & + & 85.7 & 75 & $\mathrm{p}=0.596$ \\
\hline Response & - & 14.3 & 25 & $\chi^{2}=0.514$ \\
\hline Nausea & + & 21.4 & 0 & $\mathrm{p}=0.302$ \\
\hline & - & 78.6 & 100 & $\chi^{2}=2.06$ \\
\hline Body Pain & + & 17.9 & 25 & $p=0.639$ \\
\hline & - & 82.1 & 75 & $\chi^{2}=0.203$ \\
\hline Headache & + & 25 & 25 & $\mathrm{p}=1.00$ \\
\hline & 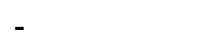 & 75 & 75 & $\chi^{2}=0.00$ \\
\hline Cough & + & 87.5 & 62.5 & $p=0.167$ \\
\hline & - & 14.3 & 37.5 & $\chi^{2}=2.141$ \\
\hline Shortness of & + & 46.4 & 37.5 & $p=0.709$ \\
\hline & - & 53.6 & 62.5 & $\chi^{2}=0.201$ \\
\hline Fever & + & 46.4 & 37.5 & $\mathrm{p}=0.691$ \\
\hline & - & 53.6 & 62.5 & $\chi^{2}=0.643$ \\
\hline
\end{tabular}


Table 2. Effect of Drug Start Time on Serum Parameters (Before)

\begin{tabular}{llll}
\hline \multirow{2}{*}{ Parameters } & \multicolumn{2}{c}{ Drug Start Time } & \multirow{2}{*}{ p-Value } \\
\cline { 2 - 3 } WBC & $0-5$ days $(\mathrm{n}=28)$ & $6-10$ days $(\mathrm{n}=8)$ & \\
\cline { 2 - 3 } Lymphocytes & $5.5 \pm 0.5$ & $5.77 \pm 0.76$ & $\mathrm{p}>0.05$ \\
HGB & $1.06 \pm 0.06$ & $1.05 \pm 0.15$ & $\mathrm{p}>0.05$ \\
HTC & $12.62 \pm 0.5$ & $13.54 \pm 0.52$ & $\mathrm{p}>0.05$ \\
Neutrophils & $41.39 \pm 0.95$ & $41.56 \pm 1.43$ & $\mathrm{p}>0.05$ \\
PLT & $4.06 \pm 0.46$ & $4.05 \pm 0.85$ & $\mathrm{p}>0.05$ \\
Creatine & $183.96 \pm 12.14$ & $160 \pm 10.98$ & $\mathrm{p}>0.05$ \\
AST & $0.94 \pm 0.04$ & $0.97 \pm 0.05$ & $\mathrm{p}>0.05$ \\
ALT & $44.71 \pm 4.9$ & $43.75 \pm 5.07$ & $\mathrm{p}>0.05$ \\
CK & $33.61 \pm 4.25$ & $41.63 \pm 9.62$ & $\mathrm{p}>0.05$ \\
CRP & $130.79 \pm 26.91$ & $205.88 \pm 73.13$ & $\mathrm{p}>0.05$ \\
BUN & $80.28 \pm 11.69$ & $72.15 \pm 29.55$ & $\mathrm{p}>0.05$ \\
D-Dimer & $29.6 \pm 2.38$ & $27.52 \pm 2.87$ & $\mathrm{p}>0.05$ \\
PCT & $1365.46 \pm 386.66$ & $886 \pm 135.59$ & $\mathrm{p}>0.05$ \\
LDH & $0.14 \pm 0.01$ & $0.16 \pm 0.03$ & $\mathrm{p}>0.05$ \\
Ferritin & $330.21 \pm 20.99$ & $401 \pm 55.74$ & $\mathrm{p}>0.05$ \\
\hline
\end{tabular}

Data are given as mean \pm standard deviation

Table 3. Effect of Drug Start Time on Serum Parameters (After)

\begin{tabular}{llll}
\hline \multirow{2}{*}{ Parameters } & \multicolumn{2}{c}{ Drug Start Time } & \multirow{2}{*}{$\mathrm{p}$-Value } \\
\cline { 2 - 4 } WBC & $0-5$ days $(\mathrm{n}=28)$ & $6-10$ days $(\mathrm{n}=8)$ & \\
\cline { 2 - 4 } Lymphocytes & $6.28 \pm 0.36$ & $11.46 \pm 4.12$ & $\mathrm{p}<0.05$ \\
HGB & $1.51 \pm 0.14$ & $1.23 \pm 0.23$ & $\mathrm{p}>0.05$ \\
HTC & $12.73 \pm 0.29$ & $12.94 \pm 0.59$ & $\mathrm{p}>0.05$ \\
Neutrophils & $40.25 \pm 0.8$ & $41.7 \pm 1.72$ & $\mathrm{p}>0.05$ \\
PLT & $4.22 \pm 0.35$ & ${ }^{*} 9.42 \pm 4.11$ & $\mathrm{p}<0.05$ \\
Creatine & $322.96 \pm 23.99$ & $269.25 \pm 28.55$ & $\mathrm{p}>0.05$ \\
AST & $0.89 \pm 0.04$ & $* 1.13 \pm 0.17$ & $\mathrm{p}<0.05$ \\
ALT & $43.14 \pm 4.75$ & $48.13 \pm 5.95$ & $\mathrm{p}>0.05$ \\
CK & $53.14 \pm 8.23$ & $65.88 \pm 18.81$ & $\mathrm{p}>0.05$ \\
CRP & $61 \pm 9.84$ & $* 304.38 \pm 161.8$ & $\mathrm{p}<0.05$ \\
BUN & $28.53 \pm 7.76$ & ${ }^{*} 74.18 \pm 39.61$ & $\mathrm{p}<0.05$ \\
D-Dimer & $30.31 \pm 3.31$ & $41.16 \pm 13.39$ & $\mathrm{p}>0.05$ \\
PCT & $964.29 \pm 236.07$ & $* 4041.13 \pm 2291.99$ & $\mathrm{p}<0.05$ \\
LDH & $0.13 \pm 0$ & $* 0.28 \pm 0.1$ & $\mathrm{p}<0.05$ \\
Ferritin & $264.21 \pm 13.82$ & $* 395.38 \pm 88.85$ & $\mathrm{p}<0.05$ \\
& $601.01 \pm 100.83$ & $753.06 \pm 204.77$ & $\mathrm{p}>0.05$ \\
\hline
\end{tabular}




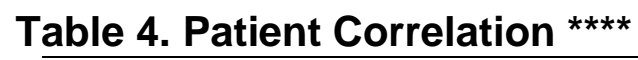

\begin{tabular}{|c|c|c|c|c|c|c|c|c|c|}
\hline & Age & DM & $\mathrm{Ht}$ & Cardiovascular & $\begin{array}{l}\text { Pulmonary } \\
\text { Disease }\end{array}$ & Cigarette & $\begin{array}{l}\text { Start } \\
\text { Time }\end{array}$ & $\begin{array}{l}\text { Latest } \\
\text { Situation }\end{array}$ & $\begin{array}{l}\text { Clinical } \\
\text { Response }\end{array}$ \\
\hline Gender & 0.140 & 0.000 & -0.126 & 0.036 & -0.287 & $0.800^{\star \star}$ & $\begin{array}{l}- \\
0.209\end{array}$ & 0.027 & -0.100 \\
\hline Age & & $\begin{array}{l}- \\
0.380^{*}\end{array}$ & $\overline{-}-551^{* *}$ & $-0.379^{*}$ & -0.159 & 0.135 & 0.029 & 0.181 & -0.011 \\
\hline DM & & & $0.365^{\star}$ & 0.139 & -0.046 & 0.129 & $\overline{-} .154$ & 0.140 & 0.086 \\
\hline $\begin{array}{l}\mathrm{Ht} \\
\text { Cardiovascular }\end{array}$ & & & & $0.412^{*}$ & $\begin{array}{l}0.006 \\
-0.161\end{array}$ & $\begin{array}{l}-0.157 \\
-0.036 \\
-0198\end{array}$ & $\begin{array}{l}0.094 \\
0.215\end{array}$ & $\begin{array}{l}-0.187 \\
-0.253\end{array}$ & $\begin{array}{l}-0.157 \\
-0.036\end{array}$ \\
\hline $\begin{array}{l}\text { pulmonary disease } \\
\text { Cigarette }\end{array}$ & & & & & & -0.198 & $\begin{array}{l}0.021 \\
- \\
0.060\end{array}$ & $\begin{array}{l}-0.253 \\
-0.027\end{array}$ & $\begin{array}{l}-0.036 \\
-0.050\end{array}$ \\
\hline $\begin{array}{l}\text { Start Time } \\
\text { Latest Situation }\end{array}$ & & & & & & & & 0.162 & $\begin{array}{l}0.120 \\
0.542^{* *}\end{array}$ \\
\hline
\end{tabular}

** Correlation is significant at the 0.01 level (2-tailed).

* Correlation is significant at the 0.05 level (2-tailed).

No statistically significant correlation was found between the time to start medication and other parameters

Table 5. The relationship between Start Time with tdsck and tdspct.

\begin{tabular}{lll}
\hline & tdsck & tdspct \\
\cline { 2 - 3 } Start Time & $0.335^{*}$ & $0.374^{*}$
\end{tabular}

* Correlation is significant at the 0.05 level (2-tailed)

A weak positive correlation was found between the time of starting the drug and tdsck $(r=0.335, p<0.05)$ and tdspct $(r=0.374, p<0)$ 